\title{
MicroRNA-100 inhibits breast cancer cell proliferation, invasion and migration by targeting FOXA1
}

\author{
HAIHUI XIE ${ }^{1,2}$, RUOBING XIAO ${ }^{1,2}$, YAOLIN HE ${ }^{1}$, LINGZHI HE ${ }^{3}$, \\ CHANGJUN XIE ${ }^{1}$, JUAN CHEN ${ }^{1}$ and YAN $\mathrm{HONG}^{3}$ \\ ${ }^{1}$ Department of Radiation Oncology, The Second Affiliated Hospital University of South China; \\ ${ }^{2}$ Clinical Research Center for Prevention and Treatment of Breast \& Thyroid Disease in Hunan Province; \\ ${ }^{3}$ Department of Preventive Medicine, Hunan Polytechnic of Environment and Biology, Hengyang, Hunan 421001, P.R. China
}

Received December 16, 2020; Accepted May 28, 2021

DOI: $10.3892 / \mathrm{ol} .2021 .13077$

\begin{abstract}
MicroRNAs (miRNAs/miRs) are highly conserved single-stranded small non-coding RNAs, which are involved in the physiological and pathological processes of breast cancer, and affect the prognosis of patients with breast cancer. The present study used the Gene Expression Omnibus (GEO)2R tool to detect miR-100 expression in breast cancer tissues obtained from GEO breast cancer-related datasets. Bioinformatics analysis revealed that miR-100 expression was downregulated in different stages, grades and lymph node metastasis stages of breast cancer, and patients with high miR-100 expression had a more favorable prognosis. Based on these analyses, Cell Counting Kit-8, wound healing and Transwell assays were performed, and the results demonstrated that overexpression of miR-100 inhibited the proliferation, migration and invasion of breast cancer cells. To verify the tumor-suppressive effect of miR-100 in breast cancer, the LinkedOmics and PITA databases were used to assess the association between miR-100 and forkhead box A1 (FOXA1). The results demonstrated that miR-100 had binding sites within the FOXA1 gene, and FOXA1 expression was negatively associated with miR-100 expression in breast cancer tissues. Similarly, a negative association was observed between miR-100 and FOXA1 expression, using the StarBase V3.0 database. The association between miR-100 and FOXA1 was further verified via reverse transcription-quantitative PCR and western blot analyses, and the dual-luciferase reporter assay. The results demonstrated that miR-100 targeted the 3'-untranslated region of FOXA1 in breast cancer cells. Furthermore, rescue experiments were performed to confirm whether miR-100 exerts its antitumor
\end{abstract}

Correspondence to: Dr Yan Hong, Department of Preventive Medicine, Hunan Polytechnic of Environment and Biology, 165 Wangcheng Road, Shigu, Hengyang, Hunan 421001, P.R. China E-mail: yanyanhongmm@163.com

Key words: microRNA-100, forkhead box A1, breast cancer, invasion, migration effects by regulating FOXA1. The results demonstrated that overexpression of FOXA1 promoted the proliferation, migration and invasion of breast cancer cells; thus, the antitumor effects of miR-100 in breast cancer were reversed following overexpression of FOXA1. Taken together, the results of the present study suggest that miR-100 inhibits the proliferation, migration and invasion of breast cancer cells by targeting FOXA1 expression. These results may provide a novel insight and an experimental basis for identifying effective therapeutic targets of high specificity for breast cancer.

\section{Introduction}

Breast cancer is one of the most common malignancies among women, whereby both its morbidity and mortality rates rank first among female malignancies, and its incidence has increased by $37 \%$ within 10 years (1). Despite the significant progress achieved in improving the diagnosis and treatment of breast cancer, metastasis remains the leading cause of mortality among patients with breast cancer (2). Thus, further in-depth studies investigating the molecular mechanisms underlying metastasis in breast cancer are urgently required to identify effective targets for breast cancer treatment.

MicroRNAs (miRNAs/miRs) are a class of highly conserved, non-coding small RNAs that are 18-25 nucleotides in length (3). The specific binding between miRNAs and the 3'-untranslated region (3'-UTR) of target mRNAs leads to the degradation or translational suppression of the target mRNA, thereby achieving the regulation of target gene expression at the post-transcriptional level (4). Previous studies have reported that miRNAs are involved in the regulation of various biological behaviors, including the proliferation, migration, invasion and metastasis of several tumor cells, by serving as either oncogenes or tumor suppressor genes. For example, miR-671-5p promotes the development and metastasis of prostate carcinoma by targeting the HNF1 homeobox A/crystallin $\alpha$ B axis (5). Furthermore, miR-490 is considered a potential biomarker and therapeutic target for cancer and other types of diseases, such as Chronic Temporal Lobe Epilepsy (6). miR-193a reduces the resistance of non-small cell lung cancer cells to cisplatin by targeting leucine rich repeat containing 1 (7). 
The present study aimed to determine the molecular mechanism of miR-100 in breast cancer. The results demonstrated that miR-100 expression was notably downregulated in different stages, subtypes and lymph node metastases stages of breast cancer. In addition, patients with high miR-100 expression had a longer survival time, suggesting the potential tumor-suppressive effects of miR-100 in breast cancer. Previous studies have also reported the differential expression of miR-100 in epithelial ovarian cancer (8) and gastric and esophageal cancer types (9), as well as its role as a biomarker for the diagnosis and treatment of breast cancer; however, to the best of our knowledge, very few studies have reported the role of miR-100 in breast cancer.

Bioinformatics analysis using the GSE45666, GSE48088, GSE44124 and GSE44899 breast cancer-related datasets from the Gene Expression Omnibus (GEO) database revealed that miR-100 expression was downregulated in breast cancer. Further in vitro functional experiments demonstrated that overexpression of miR-100 inhibited the proliferation, migration and invasion of breast cancer cells, which suggests that miR-100 may be used as a potential molecular marker and target for the diagnosis and treatment of metastatic breast cancer. The present study aimed to investigate the mechanism of miR-100 inhibiting breast cancer cell proliferation, invasion and migration.

\section{Materials and methods}

Bioinformatics analysis. The GEO database (https:/www. ncbi.nlm.nih.gov/gds) was used to obtain the following four breast cancer datasets: GSE45666, GSE48088, GSE44124 and GSE44899. Differentially expressed miRNAs in breast cancer tissues were identified using the GEO2R tool (10), with $\mid \log _{2}$ fold-change $I>1$ and $\mathrm{P}<0.05$ as the filter conditions. The prognostic significance of miR-100 in breast cancer tissues was determined using the Kaplan-Meier (KM) plotter database (http://kmplot. com/analysis/index.php? $\mathrm{p}=$ background). miR-100 expression was detected in breast, prostate, liver, lung, colon and gastric cancers, using the database of differentially expressed miRNAs in human cancers (dbDMEC; https://www. biosino.org/dbDEMC/index). The UALCAN database (http://ualcan.path.uab.edu) was used to detect miR-100 expression in breast cancers with different clinical features. The LinkedOmics database (http://www.linkedomics. org/login.php) was used to identify genes that are negatively associated with miR-100 expression in breast cancer. The PITA database (https://genie.weizmann.ac.il) was used to predict the target genes that bind to miR-100, and the intersection of genes obtained from these two databases was represented in a Venn diagram. Gene Expression Profiling Interactive Analysis (GEPIA, http://gepia.cancer-pku.cn) was used to determine the expression of the target gene, forkhead box A1 (FOXA1), in breast cancer.

Cell lines and culture. The human breast cancer cell lines, MDA-MB-231, MCF-7, HCC-1937 and ZR-75-30, and the normal human breast epithelial cell line, MCF-10A, were purchased from The Cell Bank of Type Culture Collection of The Chinese Academy of Sciences. Cells were maintained in DMEM supplemented with $10 \%$ fetal bovine serum (FBS, Gibco; Thermo Fisher Scientific, Inc.), at $37^{\circ} \mathrm{C}$ with $5 \% \mathrm{CO}_{2}$.

Cell transfection. Negative control (NC) mimic, miR-100 mimic, NC inhibitor and miR-100 inhibitor were all purchased from Guangzhou RiboBio Co., Ltd.. Due to the confidentiality policy of Guangzhou Ruibo Biological Co., Ltd., the sequences of miRNA mimics, inhibitors and NCs used in the present study are currently not available. In total, $50 \mathrm{nM}$ oligonucleotides were transfected into breast cancer cells using Lipofectamine ${ }^{\circledR} 2000$ reagent (Invitrogen; Thermo Fisher Scientific, Inc.), according to the manufacturer's instructions. Cells were collected for subsequent experimentation $48 \mathrm{~h}$ post-transfection at $37^{\circ} \mathrm{C}$.

Reverse transcription-quantitative $(R T-q) P C R$. Following transfection for $48 \mathrm{~h}$, the cell culture supernatant was discarded, cells were washed three times with PBS and total RNA was extracted using TRIzol ${ }^{\circledR}$ reagent (Invitrogen; Thermo Fisher Scientific, Inc.), according to the manufacturer's protocol. The concentration and quality of RNA was determined using a NanoDrop 2000 spectrophotometer (Thermo Fisher Scientific, Inc.). Total RNA was reverse transcribed into cDNA using a reverse transcription kit, according to the manufacturer's instructions. Reverse transcription kits include the mRNA reverse transcription kit (Japan Bio, Inc.) and the miRNA reverse transcription kit (Shanghai Guantai Biological Technology Co., Ltd.). The following conditions are used for reverse transcription: $37^{\circ} \mathrm{C}$ for $15 \mathrm{~min} ; 85^{\circ} \mathrm{C}$ for $5 \mathrm{sec} ; 4^{\circ} \mathrm{C}$ for storage. qPCR was subsequently performed to detect the expression levels of miR-100 and FOXA1, using the following reaction system: 12.5 $\mu \mathrm{l}$ SYBR Premix (Takara Bio, Inc.), $0.5 \mu \mathrm{l}$ PCR upstream primer, $0.5 \mu \mathrm{l}$ PCR downstream

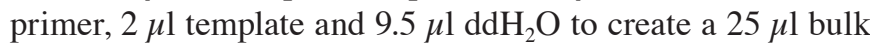
volume mixture. The following thermocycling conditions were used for qPCR: Initial denaturation at $95^{\circ} \mathrm{C}$ for $5 \mathrm{~min}$; followed by 40 cycles of denaturation at $95^{\circ} \mathrm{C}$ for $5 \mathrm{sec}$ and reaction at $60^{\circ} \mathrm{C}$ for $30 \mathrm{sec}$. The primer sequences used for qPCR are listed in Table I. Relative expression levels of miR-100 and FOXA1 were calculated using the $2^{-\Delta \Delta \mathrm{Cq}}$ method (11), and normalized to $\mathrm{U} 6$ or GAPDH as the internal controls, respectively.

Cell counting kit-8 (CCK-8) assay. The CCK-8 assay (Beyotime Institute of Biotechnology) was performed to detect cell proliferation. Briefly, cells were seeded into a 96 -well plate at a density of $5 \times 10^{3}$ cells $/ \mathrm{ml}$ and transfected at $37^{\circ} \mathrm{C}$ for $48 \mathrm{~h}$ following pre-incubation for $24 \mathrm{~h}$ at $37^{\circ} \mathrm{C}$. Following incubation for $0,24,48$ or $72 \mathrm{~h}, \mathrm{CCK}-8$ reagent was added to each well and incubated for 1-4 h. The absorbance of each well was measured at a wavelength of $450 \mathrm{~nm}$, using a microplate reader (Thermo Fisher Scientific, Inc.).

Wound healing assay. A total of three scratch lines were drawn horizontally across the underside of a 6-well plate to mark the location of the wound. Log-phase cells were collected and seeded into the 6 -well plate at a density of $1 \times 10^{6}$ cells/well, and incubated in RPMI-1640 medium containing $10 \% \mathrm{FBS}$ at $37^{\circ} \mathrm{C}$ for $24 \mathrm{~h}$ until $80 \%$ confluence was reached (12). Following transfection, three lines were scratched perpendicular to the scratch lines on the tissue culture plate using a $10 \mu \mathrm{l}$ pipette tip, 
Table I. Primer sequences used for quantitative PCR.

\begin{tabular}{ll}
\hline Primer & \multicolumn{1}{c}{ Sequence $\left(5^{\prime}-3^{\prime}\right)$} \\
\hline miR-100 & AACCCGUAGAUCCGAACUUGUG \\
GAPDH & Forward: TGCAACCGGGAAGGAAATGAA \\
& Reverse: GCATCACCCGGAGGAGAAATC \\
FOXA1 & Forward: AAGGGCATGAAACCAGCGAC \\
& Reverse: GCCTGAGTTCATGTTGCTGAC
\end{tabular}

miR, microRNA; FOXA1, forkhead box A1.

and nonadherent cells were gently washed off with PBS. The wound healing ability of the cells was visualized at 0 and $24 \mathrm{~h}$ by usinga microscope (CX23 OLYMPUS, x40) and the wound healing rate $(\%)$ was calculated using the following formula: [(Original scratch width-scratch wound at $24 \mathrm{~h}$ )/original scratch width] $\mathrm{x} 100$.

Invasion assay. The upper chambers of the Transwell plates were precoated with Matrigel (BD Biosciences) for $2 \mathrm{~h}$ at $37^{\circ} \mathrm{C}$. Log-phase breast cancer cells were subsequently collected and seeded into a 24 -well plate at a density of $2 \times 10^{5}$ cells $/ \mathrm{ml}$. Following $12 \mathrm{~h}$ of pre-incubation at $37^{\circ} \mathrm{C}$, cells were transfected for $24 \mathrm{~h}$ at $37^{\circ} \mathrm{C}$. Following transfection, cells were resuspended in serum-free cell culture medium, and a quarter of the cell $\left(1 \times 10^{5}\right.$ cells $\left./ \mathrm{ml}\right)$ suspension was suctioned and seeded into the upper chamber with serum-free of the Transwell plate, while cell culture medium supplemented with $20 \%$ FBS was added to the lower chamber. Following incubation for $24 \mathrm{~h}$, the culture medium in the upper chamber was removed and cells in the upper chamber were removed using a cotton swab. Cells in the lower chamber were fixed with $4 \%$ paraformaldehyde for $20 \mathrm{~min}$ at $4^{\circ} \mathrm{C}$ and stained with $0.1 \%$ crystal violet solution for $15 \mathrm{~min}$ at room temperature. Using microscope observation (CX23 OLYMPUS, x100) Invasive cells were counted in five randomly selected fields of view (x100).

Dual-luciferase reporter assay. The luciferase reporter gene vector (PGL3basic) was provided by Magic Biotech Co., Ltd.. Cells were seeded into a 12 -well plate at a density of $1 \times 10^{6}$ cells $/ \mathrm{ml}$, and wild-type or mutant FOXA1 3'-UTR sequences were co-transfected with miR-100 mimic, miR-100 inhibitor or their respective NCs into breast cancer cells. Following transfection using Lipofectamine ${ }^{\circledR} 2000$ Transfection Reagent (Invitrogen; Thermo Fisher Scientific, Inc.) for $48 \mathrm{~h}$ at $37^{\circ} \mathrm{C}$, relative firefly and Renilla luciferase activities within each group of cells were detected using the Dual Luciferase Reporter Gene Assay kit (Promega Corporation). Firefly luciferase activity was normalized to Renilla luciferase activity.

Western blotting. Following transfection for $48 \mathrm{~h}$, the cell culture supernatant was discarded and cells were washed three times with cold PBS. Total protein was extracted from cells using cell lysis buffer (Beyotime Institute of Biotechnology) on ice for $30 \mathrm{~min}$. Following centrifugation at 12,000 x $\mathrm{g}$ for $15 \mathrm{~min}$ at $4^{\circ} \mathrm{C}$, the supernatant was discarded and total protein was obtained. Protein concentration was measured using the BCA protein assay kit (Beyotime Institute of Biotechnology) and $30 \mu \mathrm{g}$ protein/lane was separated via 10\% SDS-PAGE. The separated proteins were subsequently transferred onto PVDF membranes and blocked with 5\% non-fat milk for $2 \mathrm{~h}$ at room temperature. The membranes were washed with $1 \%$ TBS-T (0.05\%-Tween 20$)$ and incubated with primary antibodies against GAPDH (cat. no. A19056; Abclonal Biotech Co., Ltd.) and FOXA1 (cat. no. A15278; Abclonal Biotech Co., Ltd.) overnight at $4^{\circ} \mathrm{C}$ (both 1:1,000). Following the primary incubation, membranes were incubated with a goat anti-mouse secondary antibody (1:10,000; cat. nos. AS003 and AS014; ABclonal Biotech, Co., Ltd.) at $37^{\circ} \mathrm{C}$ for $2 \mathrm{~h}$. Protein bands were visualized using an ECL solution (MilliporeSigma) and densitometric analysis was performed using ImageJ software (v1.8.0.112; National Institutes of Health), with GAPDH as the internal loading control.

Statistical analysis. Statistical analysis was performed using GraphPad 8.0 software (GraphPad Software, Inc.). All experiments were performed in triplicate and data are presented as the mean \pm SD. Paired or unpaired Svtudent's t-test was used to compare differences between two groups, while one-way ANOVA followed by Tukey's post hoc test was used to compare differences between multiple groups. Two-way ANOVA followed by Sidak post hoc test was used for the dual-luciferase reporter assay. The LinkedOmics database (http://www.linkedomics.org/login.php) and StarBase V3.0 (http://starbase.sysu.edu.cn/index.php) were used to assess the correlation between FOXA1 and miR-100 expression. $\mathrm{P}<0.05$ was considered to indicate a statistically significant difference.

\section{Results}

miR-100 expression and prognostic significance in breast cancer. The GSE45666, GSE48088, GSE44124 and GSE44899 datasets were analyzed using the GEO2R tool to identify differentially expressed miRNAs in breast cancer. The results revealed that $19,25,20$ and 13 miRNAs were upregulated, and 268, 30, 28 and 42 miRNAs were downregulated in each dataset, respectively. According to the Venn diagrams, only one miRNA was upregulated, and three miRNAs were downregulated in breast cancer (Fig. 1A). Following a literature search and subsequent analysis, the downregulated miRNA, miR-100, was selected as the focus of the present study. Based on the data retrieved from the KM plotter database, patients with breast cancer with high miR-100 expression had a more favorable prognosis (Fig. 1B). The samples from the GEO database were further analyzed using the dbDMEC, which revealed that miR-100 expression was downregulated in breast cancer. In particular, miR-100 expression was downregulated in high grade breast cancer compared with low grade breast cancer, and relatively downregulated in metastatic breast cancer cases (Fig. 1C). Samples from The Cancer Genome Atlas database (TCGA, https://portal.gdc.cancer.gov) were analyzed using the UALCAN database. The results demonstrated that miR-100 expression was downregulated in different stages and grades of breast cancer, tumor histological types, menstrual phases and lymph node metastasis stages. The results demonstrated that miR-100 is expressed at low levels in primary breast cancer 
A

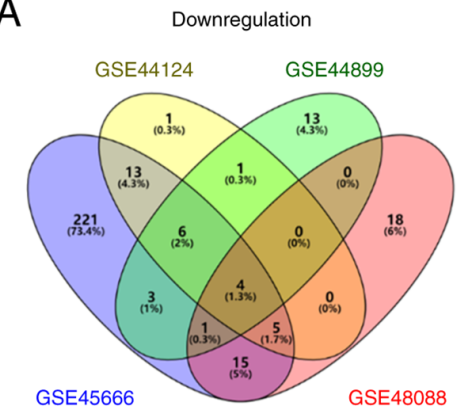

Upregulated

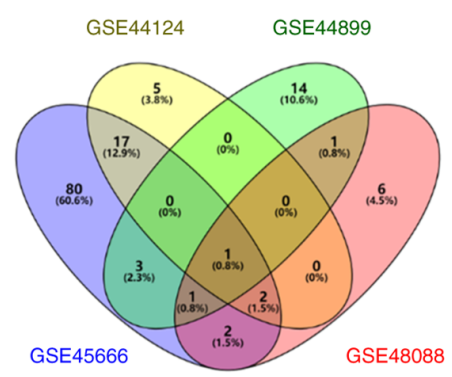

B

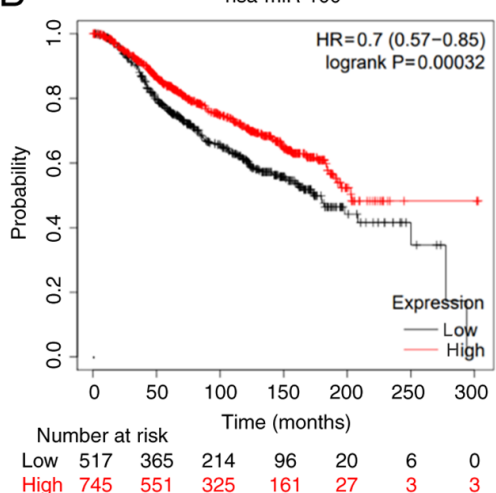

C

Differential expression profile in cancer vs. normal
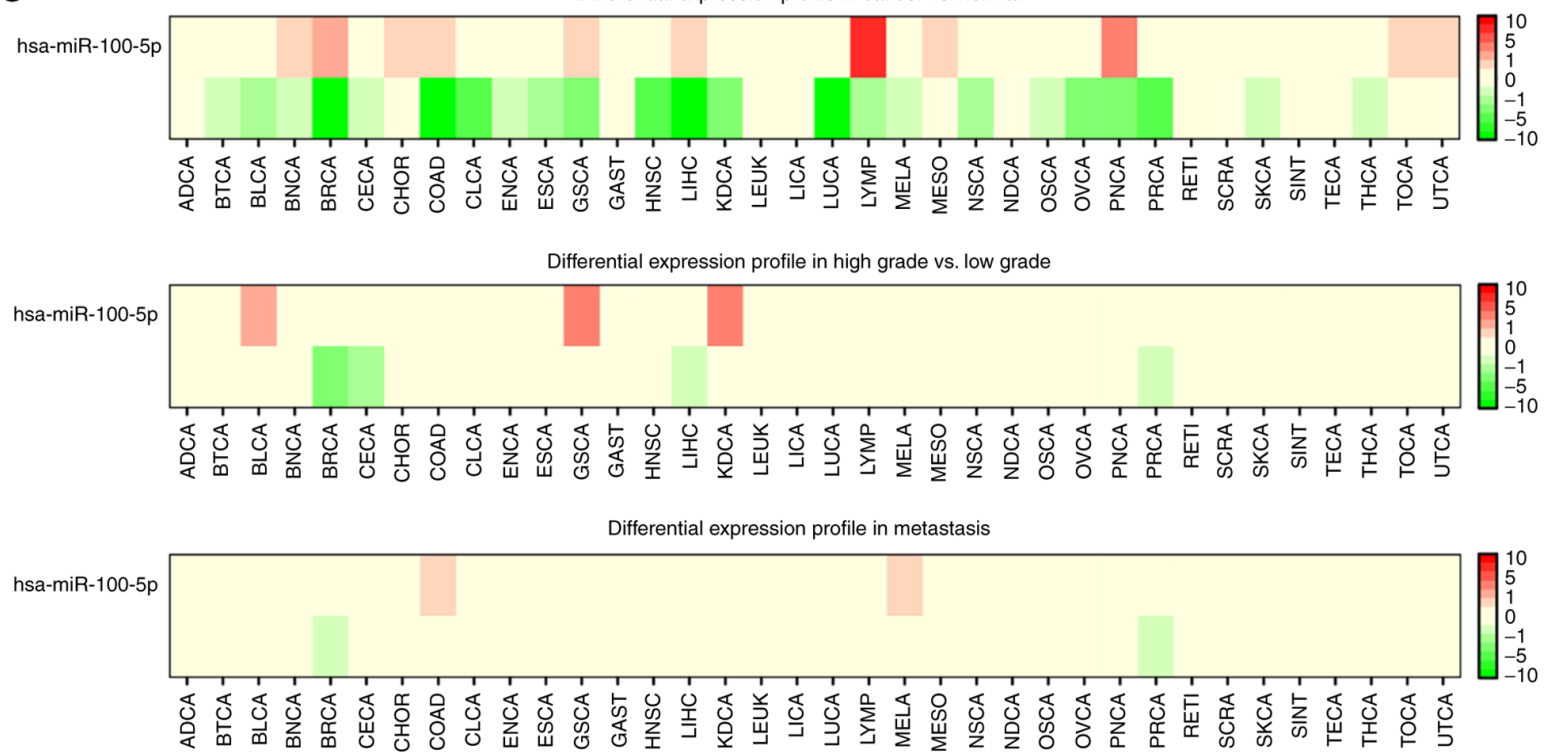

Figure 1. miR-100 expression and prognostic significance in breast cancer. (A) Venn diagrams were used to display differentially expressed miRNAs identified from the GSE45666, GSE48088, GSE44124 and GSE44899 datasets. (B) The Kaplan-Meier plotter database was used to determine the prognostic significance of miR-100 in breast cancer. (C) The database of differentially expressed miRNAs in human cancers was used to detect miR-100 expression in breast cancer. $\mathrm{miR} / \mathrm{miRNA}$, microRNA.

(Fig. 2A), breast cancer staging (Fig. 2B), lymphatic metastatic breast cancer (Fig. 2C), menopausal patients (Fig. 2D), breast cancer subclasses (Fig. 2E) and the histological classification of breast cancer (Fig. 2F). Taken together, these results suggest that miR-100 expression is downregulated in breast cancer and associated with the metastasis and staging of breast cancer.

miR-100 expression in breast cancer cells. miR-100 expression was detected in the four breast cancer cell lines (MCF-7, HCC-1937, ZR-76-30 and MDA-MB-231) and the human normal breast epithelial cell line, MCF-10A. The results demonstrated that miR-100 expression was downregulated in the breast cancer cell lines compared with the normal cells (Fig. 3A). Among the cell lines, miR-100 expression was highest in MCF-7 cells and lowest in MDA-MB-231 cells. Thus, MCF-7 cells were selected as the model to knockdown miR-100, while MDB-MB-231 cells were selected as the model to overexpress miR-100. miR-100 knockdown in MCF-7 cells decreased miR-100 expression by 0.42 -fold (Fig. 3B), while overexpression of miR-100 increased miR-100 expression by 26.3-fold in MDA-MB-231 cells (Fig. 3C).
Overexpression of miR-100 inhibits the proliferation, invasion and migration of breast cancer cells. As presented in Fig. 4A, overexpression of miR-100 significantly inhibited the proliferation of MDA-MB-231 cells $(\mathrm{P}<0.0001)$, while miR-100 knockdown significantly promoted the proliferation of MCF-7 cells $(\mathrm{P}<0.001)$. Similarly, the results of the Transwell and wound healing assays demonstrated that overexpression of miR-100 decreased the invasive and migratory abilities of MDA-MB-231 cells $(\mathrm{P}<0.05)$, the effects of which were reversed following miR-100 knockdown in MCF-7 cells $(\mathrm{P}<0.01$ and $\mathrm{P}<0.05$, respectively). Collectively, these results suggest that overexpression of miR-100 may inhibit the proliferation, invasion and migration of breast cancer cells.

Prediction of miR-100 target genes. Overexpression of miR-100 inhibited the proliferation, migration and invasion of breast cancer cells. Thus, the present study aimed to determine its underlying molecular mechanism. Given that miRNA mainly functions by targeting the 3'-UTR of target mRNA (13), the LinkedOmics database was used to identify genes negatively associated with miR-100 expression in breast cancer (Fig. 5A). In addition, the target genes of miR-100 were 
A

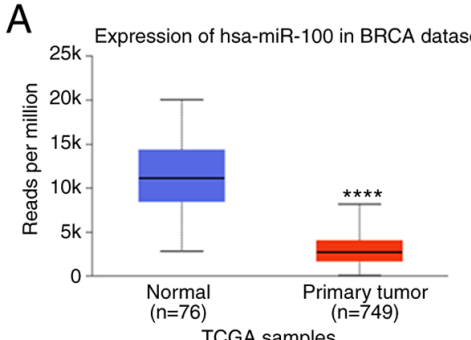

D

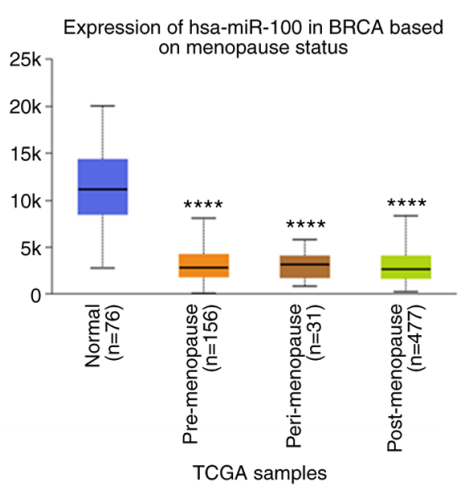

$\mathrm{B}$

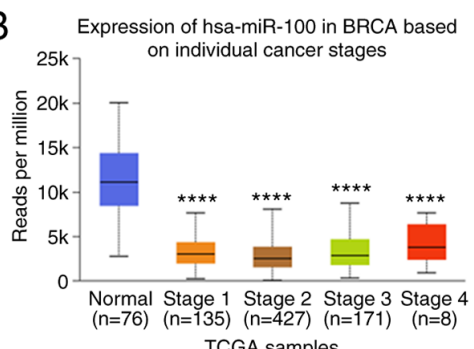

E

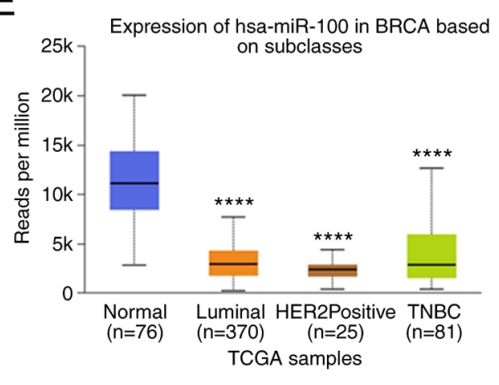

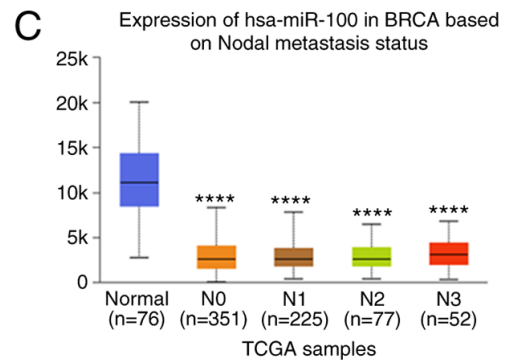

$\mathrm{F}$

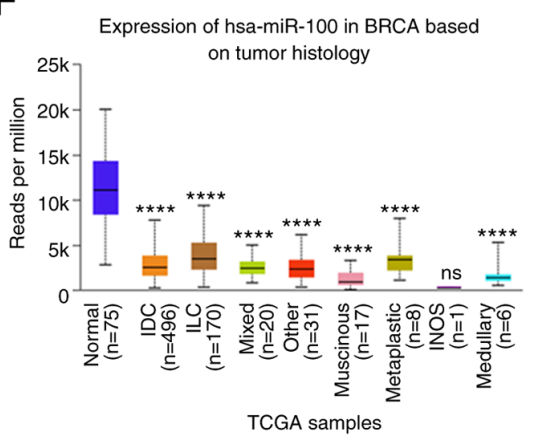

Figure 2. miR-100 expression in breast cancers with different clinical features. (A) hsa-mir-100 expression in the BRCA dataset. (B) hsa-mir-100 expression in BRCA based on individual cancer stages. (C) hsa-mir-100 expression in BRCA based on nodal metastasis status. (D) hsa-mir-100 expression in BRCA based on Menopause status. (E) hsa-mir-100 expression in BRCA based on subclasses. (F) hsa-mir-100 expression in BRCA based on Tumor histology. ${ }^{* * * * *} \mathrm{P}<0.0001$ vs. normal group. miR, microRNA; TCGA, The Cancer Genome Atlas; HER2, human epidermal growth factor receptor 2; TNBC, triple-negative breast cancer; IDC, invasive ductal carcinoma; ILC, invasive lobular carcinoma; INOS, inducible nitric oxide synthase.

A

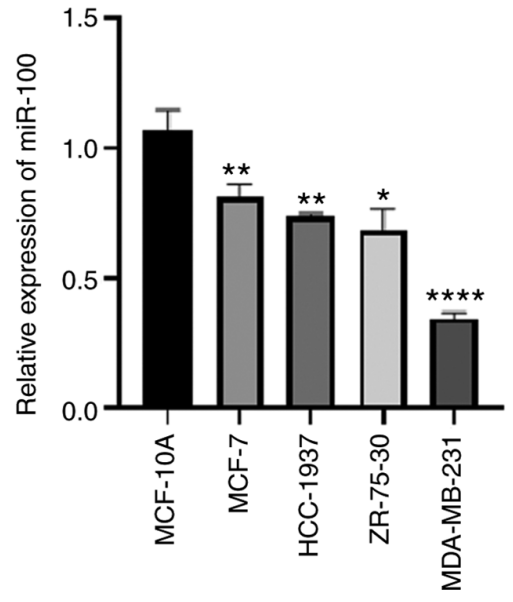

B

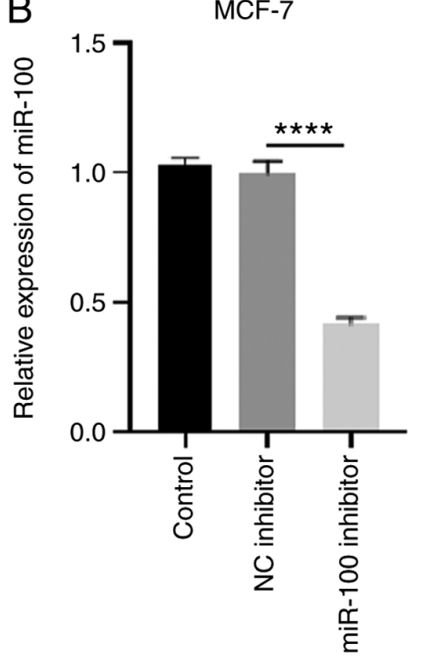

C

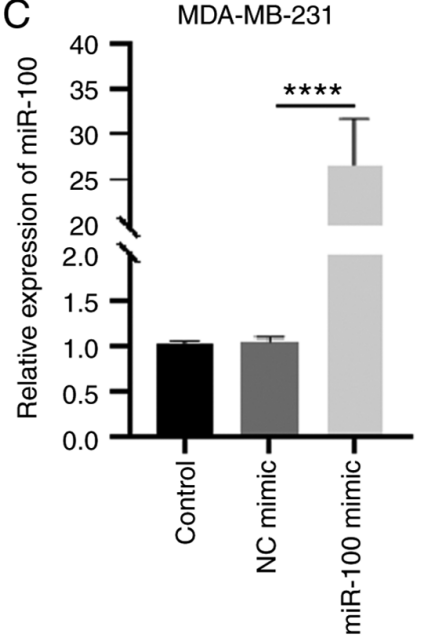

Figure 3. miR-100 expression, and transfection efficiency of miR-100 inhibitors and mimic in breast cancer cells. (A) miR-100 expression levels in breast cancer cells and human normal breast epithelial cells. (B) Knockdown efficiency of miR-100 in MCF-7 cells. (C) Overexpression efficiency of miR-100 in MBA-MB-231 cells. ${ }^{*} \mathrm{P}<0.05,{ }^{* *} \mathrm{P}<0.01$ vs. MCF-10A cells; ${ }^{* * * * * *} \mathrm{P}<0.0001$. miR, microRNA; NC, negative control.

predicted using the PITA database. The intersection of the target genes from both databases identified FOXA1 as a target gene of miR-100 (Fig. 5B). According to the LinkedOmics database, the correlation coefficient between FOXA1 and miR-100 was -0.2621 ( $\mathrm{P}<0.0001$; Fig. 5C). The correlation between miR-100 and FOXA1 expression was further verified using StarBase V3.0, and the correlation coefficient was -0.134 $(\mathrm{P}<0.0001$; Fig. 5D). FOXA1 expression in breast cancer tissues was analyzed using the GEPIA database, and the results demonstrated that FOXA1 expression was upregulated in breast cancer tissues compared with adjacent normal tissues (Fig. 5E). Taken together, these results suggest that FOXA1 may be a potential target gene of miR-100.

miR-100 targets the 3'-UTR of FOXA1. The prediction of the correlation between miR-100 and FOXA1 expression was validated by in vitro experiments. RT-qPCR and western blot analyses demonstrated that overexpression of miR-100 decreased both the mRNA and protein expression levels of FOXA1, while miR-100 knockdown increased the mRNA 

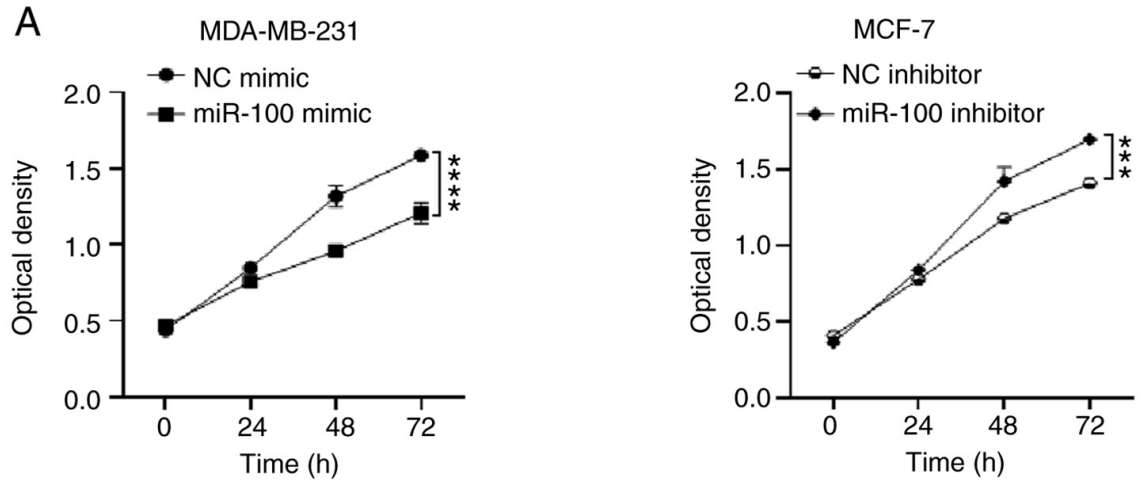

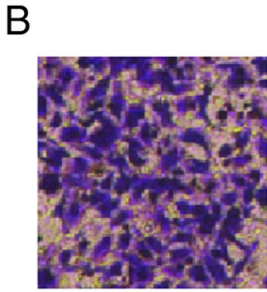

NC mimic

C
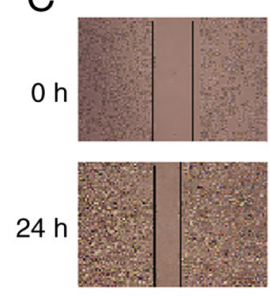

NC mimic

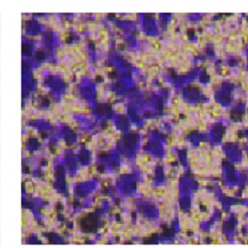

miR-100 mimic
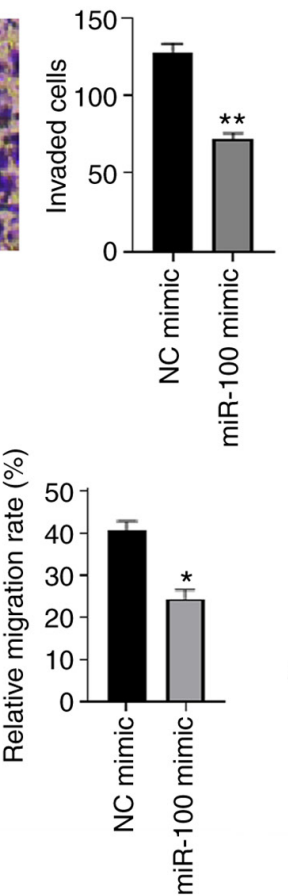

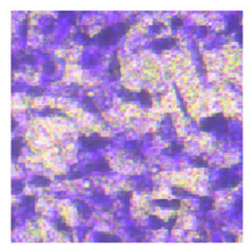

NC inhibitor

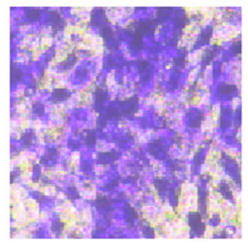

miR-100 inhibitor
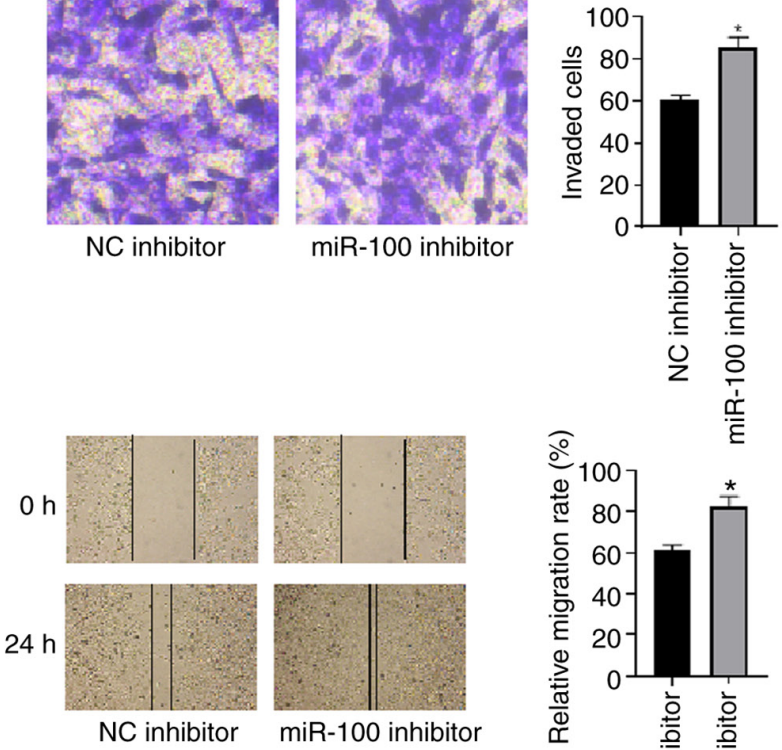

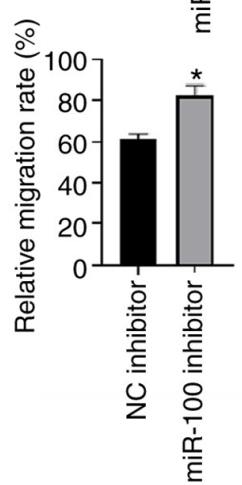

Figure 4. Effects of overexpressing miR-100 on the proliferation, invasion and migration of breast cancer cells. (A) The Cell Counting Kit-8 assay was performed to assess the effect of overexpressing miR-100 on the proliferation of breast cancer cells. (B) The Transwell assay was performed to assess the effect of overexpressing miR-100 on the invasion of breast cancer cells. (C) The wound healing assay was performed to assess the effect of overexpressing miR-100 on the migration of breast cancer cells. ${ }^{*} \mathrm{P}<0.05,{ }^{* *} \mathrm{P}<0.01,{ }^{* *} \mathrm{P}<0.001,{ }^{* * * *} \mathrm{P}<0.0001$ vs. the NC mimic group. miR, microRNA; NC, negative control.

and protein expression levels of FOXA1 (Fig. 6A and B). The results of the dual-luciferase reporter assay demonstrated that relative luciferase activity was notably reduced following co-transfection of miR-100 mimic with wild-type FOXA1 3'-UTR into MDA-MB-231; the opposite result was observed in MCF-7 cells. ( $\mathrm{P}<0.001$ and $\mathrm{P}<0.01$, respectively; Fig. 6C). However, relative luciferase activity did not change following co-transfection of miR-100 mimic or miR-100 inhibitor with mutant FOXA1 3'-UTR into MDA-MB-231 or MCF-7 cells, respectively. Collectively, these results suggest that miR-100 may target the 3'-UTR of FOXA1.

miR-100 exerts antitumor effects by targeting FOXA1. To confirm whether miR-100 exerts antitumor effects by regulating FOXA1 expression, rescue experiments were performed. As presented in Fig. 7A, overexpression of FOXA1 promoted the proliferation of MDA-MB-231 cells $(\mathrm{P}<0.05)$, while cell proliferation was partially inhibited under the combined action of miR-100 mimic and FOXA1 overexpression plasmid
$(\mathrm{P}<0.001)$. Similarly, overexpression of FOXA1 promoted the invasive and migratory abilities of MDA-MB-231 cells $(\mathrm{P}<0.0001$ and $\mathrm{P}<0.001$, respectively), while the invasive and migratory abilities of MDA-MB-231 cells decreased following simultaneous transfection with miR-100 mimic and FOXA1 overexpression plasmid $(\mathrm{P}<0.001$ and $\mathrm{P}<0.01$, respectively; Fig. 7B-D). Taken together, these results suggest that overexpression of FOXA1 may promote the proliferation, invasion and migration of breast cancer cells, inhibiting the effects of overexpressing miR-100 on the proliferation, invasion and migration of breast cancer cells, which explains how miR-100 exerts its antitumor effects in breast cancer via FOXA1.

\section{Discussion}

miRNAs have been reported to play important regulatory roles in tumorigenesis and cancer progression, and are involved in various biological processes, such as cell proliferation, apoptosis, differentiation, invasion, metastasis and drug 
A

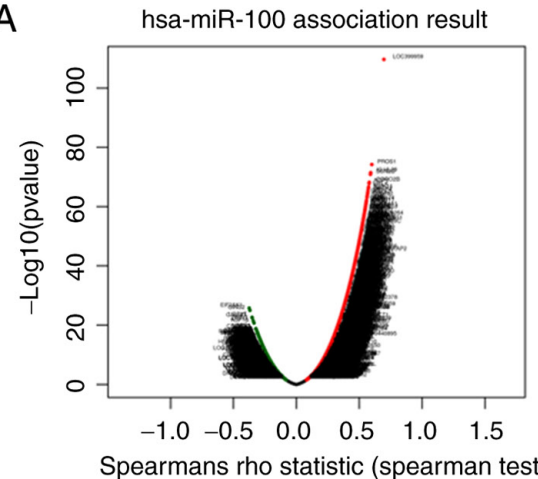

B

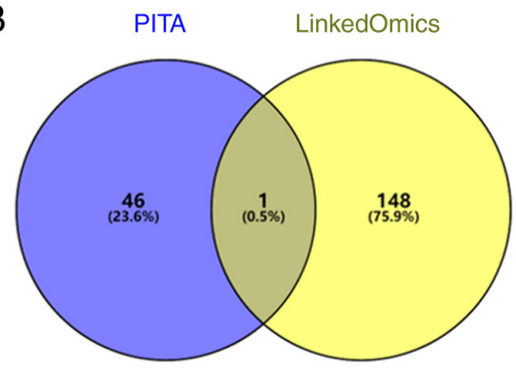

C

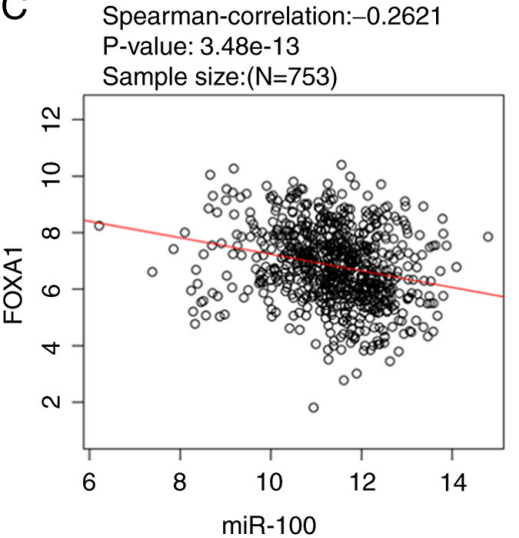

D

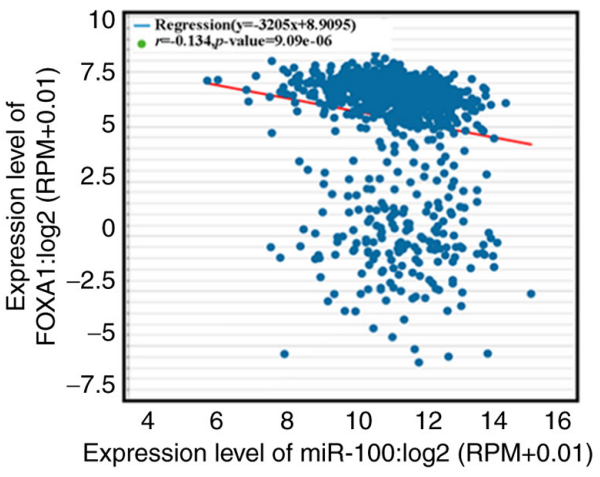

E

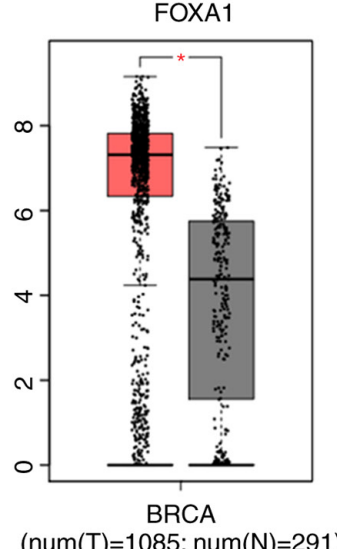

Figure 5. Bioinformatics analysis on identifying target genes of miR-100. (A) Volcano plot based on the LinkedOmics database depicting genes positively and negatively correlated with miR-100 in breast cancer. (B) Venn diagram was used to represent the intersection of targets genes of miR-100 predicted using the PITA database and the genes negatively correlated with miR-100 obtained from the LinkedOmics database. (C) The LinkedOmics database was used to determine the correlation between miR-100 and FOXA1 expression in breast cancer. (D) The StarBase V3.0 database was used to determine the correlation between miR-100 and FOXA1 expression in breast cancer. (E) The Gene Expression Profiling Interactive Analysis database was used to detect FOXA1 expression in breast cancer. "P<0.05. miR, microRNA; FOXA1, forkhead box A1; T, tumor; $\mathrm{N}$, normal.
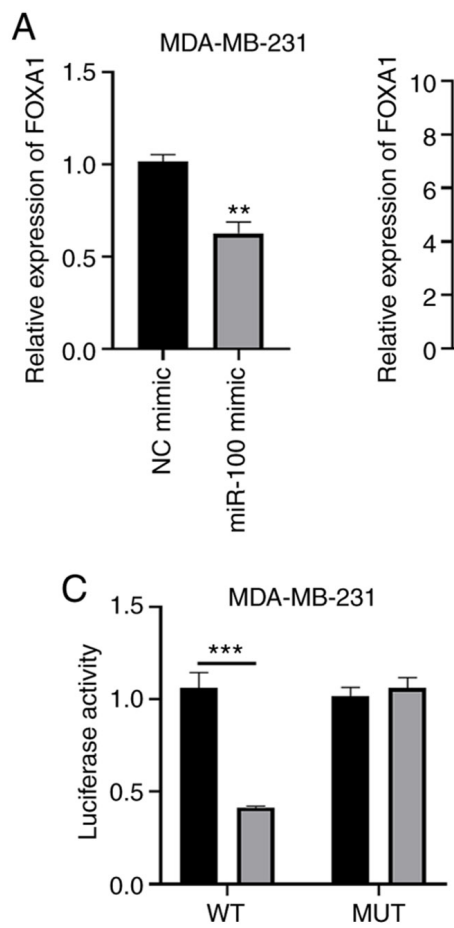

MCF-7
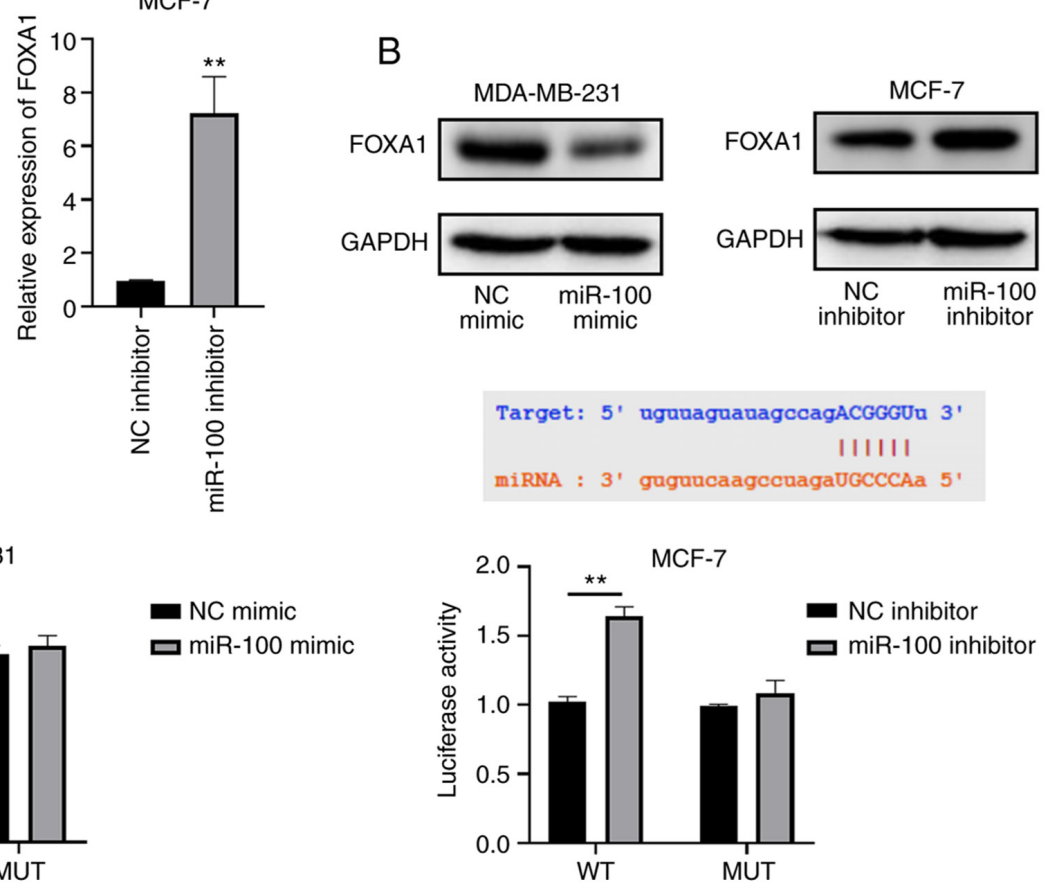

Figure 6. miR-100 targets FOXA1. (A) Reverse transcription-quantitative PCR analysis was performed to assess the effect of altering miR-100 expression on FOXA1 mRNA expression. (B) Western blot analysis was performed to assess the effect of altering miR-100 expression on FOXA1 protein expression. (C) The dual-luciferase reporter assay demonstrated that miR-100 targeted the 3 '-untranslated region of FOXA1. ${ }^{* *} \mathrm{P}<0.01,{ }^{* * * *} \mathrm{P}<0.001$ vs. the NC or miR-100 mimic groups. miR, microRNA; FOXA1, forkhead box A1; NC, negative control; WT, wild-type; MUT, mutant. 

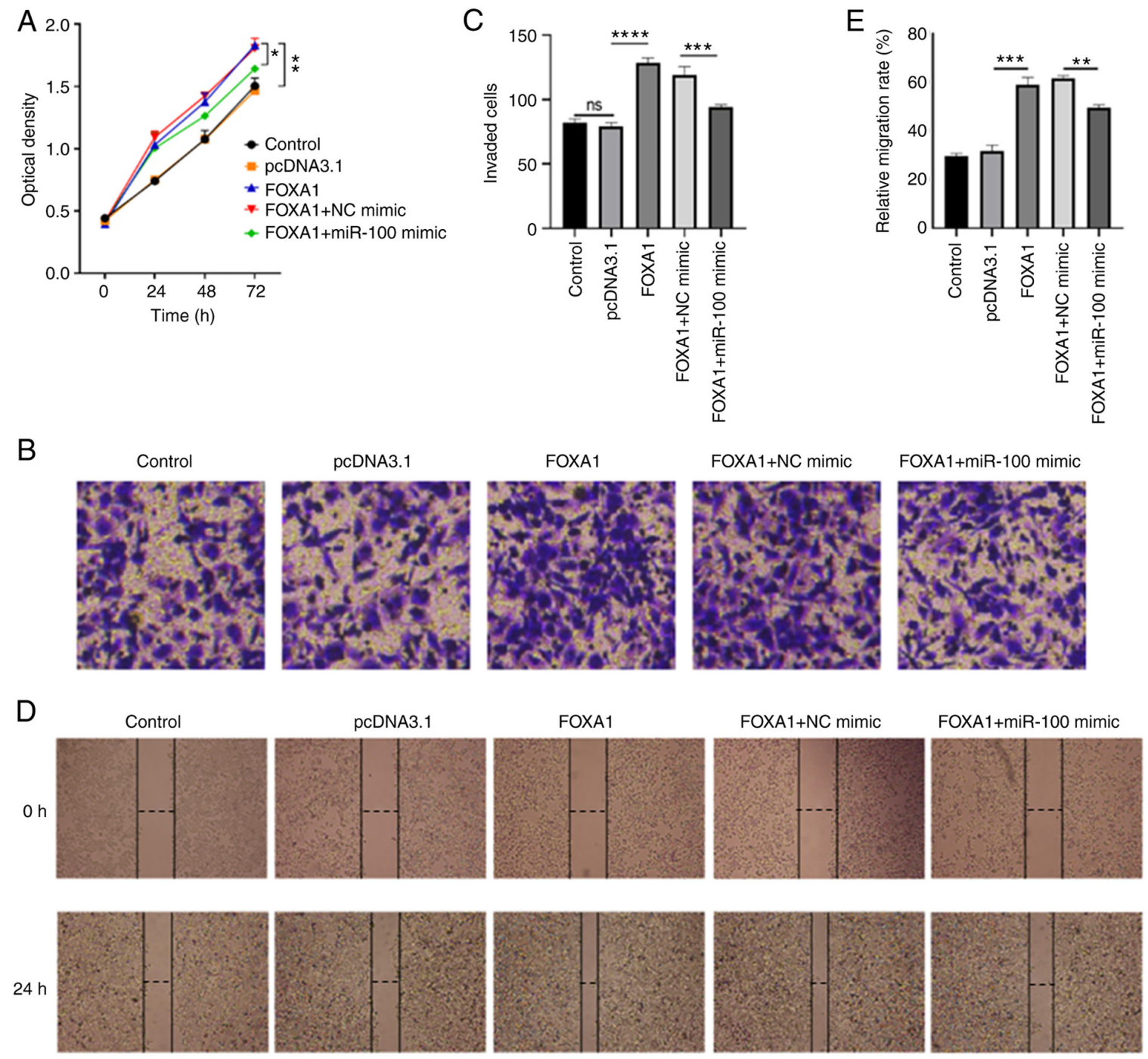

Figure 7. Overexpression of miR-100 inhibits the proliferation, invasion and migration of breast cancer cells. (A) The Cell Counting Kit-8 assay demonstrated that overexpression of FOXA1 reversed the effects of overexpressing miR-100 on the proliferation of MDA-MB-231 cells. (B) The Transwell assay demonstrated that overexpression of FOXA1 reversed the effects of overexpressing miR-100 on the invasion of MDA-MB-231 cells. (C) The Transwell assay detected invasion of cells in each group. (D) The wound healing assay revealed that overexpression of FOXA1 reversed the effects of overexpressing miR-100 on the migration of MDA-MB-231 cells. (E) The wound healing assay detected the lateral migration of cells in each group. The wound healing assay revealed that overexpression of FOXA1 reversed the effects of overexpressing miR-100 on the migration of MDA-MB-231 cells. ${ }^{*} \mathrm{P}<0.05 ;{ }^{* * *} \mathrm{P}<0.01 ;{ }^{* * * *} \mathrm{P}<0.001 ;{ }^{* * * * *} \mathrm{P}<0.0001$. miR, microRNA; FOXA1, forkhead box A1; NC, negative control.

resistance $(14,15)$. The present study analyzed GEO datasets containing breast cancer tissues and adjacent normal tissues, and the results demonstrated that miR-100 expression was notably downregulated in breast cancer tissues, and the overall survival time of patients with breast cancer with high miR-100 expression was longer than those with low miR-100 expression. In addition, the results of the CCK-8, wound healing and Transwell assays demonstrated that overexpression of miR-100 inhibited the proliferation, migration and invasion of breast cancer cells, respectively.

miR-100 has been reported to play an important role in diseases, including atherosclerosis (16), acute lung injury (17), pulmonary hypertension (18), childhood acute lymphoblastic leukemia (19) and osteoarthritis of the temporomandibular joint (20). In addition, miR-100 has been suggested to serve as a diagnostic and therapeutic biomarker in epithelial ovarian cancer (8) and gastric and esophageal cancers (9). miR-100 decreases glioblastoma growth by targeting SWI/SNF related, matrix associated, actin dependent regulator of chromatin, subfamily a, member 5 and erb-b2 receptor tyrosine kinase 3 (21), and suppresses tumor growth in cervical cancer by downregulating SATB homeobox 1 expression and regulating the AKT/mTOR signaling pathway and epithelial-to-mesenchymal transition (22). The role of miR-100 in breast cancer has also been investigated in several studies. For example, miR-100 expression has been reported to be downregulated in breast cancer, which inhibits the proliferation and viability of breast cancer cells by regulating insulin like growth factor 2 expression (23). In addition, miR-100 blocks the $\mathrm{Wnt} / \beta$-catenin signaling pathway and inhibits the migration and invasion of breast cancer cells by targeting frizzled class receptor 8 (24). The results of the present study demonstrated that miR-100 expression was downregulated in breast cancer, which inhibited the proliferation, migration and invasion of breast cancer cells. These results are consistent with previous findings, validating the results presented here. 
The present study aimed to determine the molecular mechanism by which miR-100 exerts antitumor effects in breast cancer. In most cases, miR-100 functions by regulating target gene expression by binding to the 3'-UTRs of its target genes (25). Thus, bioinformatics analysis was performed using the LinkedOmics database to identify genes that are negatively associated with miR-100 expression in breast cancer. In addition, the PITA database was used to identify target genes that bind with miR-100. The intersection of the results of these two databases identified FOXA1 as a target gene. Analysis using the GEPIA database revealed that FOXA1 expression was upregulated in breast cancer. RT-qPCR and western blot analyses, and the results of the dual-luciferase reporter assay verified that miR-100 was able to target the 3'-UTR of FOXA1 and negatively regulate FOXA1 expression. In vitro cell functional experiments also demonstrated that FOXA1 promoted the proliferation, migration and invasion of breast cancer cells. Upregulated FOXA1 expression not only predicts late recurrence in estrogen-positive and human epidermal growth factor receptor 2 (HER2)-negative patients with breast cancer (26), but also induces enhancer programming and hypoxia inducible factor- $2 \alpha$-dependent transcriptional programs during the treatment of endocrine-resistant metastatic breast cancer (27). In a previous study, FOXA1 expression was reported to be upregulated in breast cancer, which was positively associated with estrogen receptor, progesterone receptor, HER2 and nodal statuses (28). This suggests that FOXA1 may play a key role in breast cancer, and these findings are consistent with the results of the present study.

In the present study, the effects of miR-100 and FOXA1 in breast cancer and the targeting association between the two were verified. Whether miR-100 regulates FOXA1 expression to inhibit the proliferation, invasion and migration of breast cancer cells was also investigated using rescue experiments. Notably, the association between miR-100 and FOXA1 expression in breast cancer has rarely been reported, expect for a study that investigated the role of miR-100 as a predictor of endocrine responsiveness and prognosis in hormone receptor-positive/HER2-negative patients with breast cancer (29). However, the present study focused on all subtypes of breast cancer to determine the effect of miR-100 on breast cancer migration and invasion.

In the present study, bioinformatics analysis and experimental verification were performed to analyze the role and potential molecular mechanism of miR-100 in breast cancer. The results provide potential targets for clinical treatment and diagnosis.

In conclusion, the results of the present study demonstrated that miR-100 expression was downregulated in breast cancer, and overexpression of miR-100 inhibited the proliferation, migration and invasion of breast cancer cells. Conversely, as a target gene of miR-100, overexpression of FOXA1 promoted the proliferation, migration and invasion of breast cancer cells. Taken together, these results suggest that miR-100 and FOXA1 play important roles in breast cancer, and that the miR-100/FOXA1 signaling axis may represent a therapeutic target for breast cancer.

\section{Acknowledgements}

Not applicable.

\section{Funding}

No funding was received.

\section{Availability of data and materials}

The datasets used and/or analyzed during the current study are available from the corresponding author upon reasonable request.

\section{Authors' contributions}

RX, HX and CX performed bioinformatics analysis and selected the key molecules of the study. HX, YH and YLH designed and analyzed the in vitro assays. JC and LH designed and analyzed the mouse experiment. YH drafted and revised the initial manuscript. HX and YH confirmed the authenticity of all the raw data. All authors provided their opinions on the article and data. All authors have read and approved the final manuscript.

\section{Ethics approval and consent to participate}

Not applicable.

\section{Patient consent for publication}

Not applicable.

\section{Competing interests}

The authors declare that they have no competing interests.

\section{References}

1. Yin X, Wang P, Yang T, Li G, Teng X, Huang W and Yu H: Identification of key modules and genes associated with breast cancer prognosis using WGCNA and ceRNA network analysis. Aging (Albany NY) 13: 2519-2538, 2020.

2. Fahad Ullah M: Breast cancer: Current perspectives on the disease status. Adv Exp Med Biol 1152: 51-64, 2019.

3. Correia de Sousa M, Gjorgjieva M, Dolicka D, Sobolewski C and Foti M: Deciphering miRNAs' action through miRNA editing. Int J Mol Sci 20: 6249, 2019.

4. Chen M, Chen C, Luo H, Ren J, Dai Q, Hu W, Zhou K, Tang X and Li X: MicroRNA-296-5p inhibits cell metastasis and invasion in nasopharyngeal carcinoma by reversing transforming growth factor- $\beta$-induced epithelial-mesenchymal transition. Cell Mol Biol Lett 25: 49, 2020.

5. Zhu Z, Luo L, Xiang Q, Wang J, Liu Y, Deng Y and Zhao Z: MiRNA-671-5p promotes prostate cancer development and metastasis by targeting NFIA/CRYAB axis. Cell Death Dis 11: 949, 2020.

6. Vinchure OS and Kulshreshtha R: miR-490: A potential biomarker and therapeutic target in cancer and other diseases. J Cell Physiol 236: 3178-3193, 2021.

7. Wu H, Mu X, Liu L, Wu H, Hu X, Chen L, Liu J, Mu Y, Yuan F, Liu W and Zhao Y: Bone marrow mesenchymal stem cells-derived exosomal microRNA-193a reduces cisplatin resistance of non-small cell lung cancer cells via targeting LRRC1. Cell Death Dis 11: 801, 2020.

8. Pan C, Stevic I, Müller V, Ni Q, Oliveira-Ferrer L, Pantel K and Schwarzenbach H: Exosomal microRNAs as tumor markers in epithelial ovarian cancer. Mol Oncol 12: 1935-1948, 2018.

9. Jamali L, Tofigh R, Tutunchi S, Panahi G, Borhani F, Akhavan S, Nourmohammadi P, Ghaderian SMH, Rasouli M and Mirzaei H: Circulating microRNAs as diagnostic and therapeutic biomarkers in gastric and esophageal cancers. J Cell Physiol 233: 8538-8550, 2018. 
10. Feng $\mathrm{H}, \mathrm{Gu} \mathrm{ZY}$, Li Q, Liu QH, Yang XY and Zhang JJ: Identification of significant genes with poor prognosis in ovarian cancer via bioinformatical analysis. J Ovarian Res 12: 35, 2019.

11. Livak KJ and Schmittgen TD: Analysis of relative gene expression data using real-time quantitative PCR and the 2(-Delta Delta C(T)) method. Methods 25: 402-408, 2001.

12. Wössner N, Alhalabi Z, González J, Swyter S, Gan J, Schmidtkunz K, Zhang L, Vaquero A, Ovaa H, Einsle O, et al: Sirtuin 1 inhibiting thiocyanates (S1th)-a new class of isotype selective inhibitors of $\mathrm{NAD}^{+}$dependent lysine deacetylases. Front Oncol 10: 657, 2020.

13. Rouleau S, Glouzon JS, Brumwell A, Bisaillon $M$ and Perreault JP: 3'UTR G-quadruplexes regulate miRNA binding. RNA 23: 1172-1179, 2017.

14. Chen L, Heikkinen L, Wang C, Yang Y, Sun H and Wong G: Trends in the development of miRNA bioinformatics tools. Brief Bioinform 20: 1836-1852, 2019.

15. Cao T and Zhen XC: Dysregulation of miRNA and its potential therapeutic application in schizophrenia. CNS Neurosci Ther 24: 586-597, 2018.

16. Linna-Kuosmanen S, Bosch V, Moreau P, Moreau PR, Bouvy-Liivrand M, Niskanen $H$, Kansanen E, Kivelä A, Hartikainen J, Hippeläinen M, et al: NRF2 is a key regulator of endothelial microRNA expression under proatherogenic stimuli. Cardiovasc Res 117: 1339-1357, 2021

17. Chen WX, Zhou J, Zhou SS, Zhang YD, Ji TY, Zhang XL, Wang SM, Du T and Ding DG: Microvesicles derived from human Wharton's jelly mesenchymal stem cells enhance autophagy and ameliorate acute lung injury via delivery of miR-100. Stem Cell Res Ther 11: 113, 2020.

18. Wang J, Hu L, Huang H, Yu Y, Wang J, Yu Y, Li K, Li Y, Tian T and Chen F: CAR (CARSKNKDC) peptide modified ReNcell-derived extracellular vesicles as a novel therapeutic agent for targeted pulmonary hypertension therapy. Hypertension 76 : 1147-1160, 2020.

19. Rashed WM, Hamza MM, Matboli M and Salem SI: MicroRNA as a prognostic biomarker for survival in childhood acute lymphoblastic leukemia: A systematic review. Cancer Metastasis Rev 38: 771-782, 2019.

20. Luo P, Jiang C, Ji P, Wang $M$ and Xu J: Exosomes of stem cells from human exfoliated deciduous teeth as an anti-inflammatory agent in temporomandibular joint chondrocytes via miR-100-5p/mTOR. Stem Cell Res Ther 10: 216, 2019.

21. Alrfaei BM, Clark P, Vemuganti R and Kuo JS: MicroRNA miR-100 decreases glioblastoma growth by targeting SMARCA5 and ErbB3 in tumor-initiating cells. Technol Cancer Res Treat 19: $1533033820960748,2020$.
22. Huang C, Qin X, Zhao N, Jin H, Zhang S and Yang $H$ MicroRNA-100 functions as a tumor suppressor in cervical cancer via downregulating the SATB1 expression and regulating AKT/mTOR signaling pathway and epithelial-to-mesenchymal transition. Oncol Lett 20: 1336-1344, 2020.

23. Gebeshuber CA and Martinez J: miR-100 suppresses IGF2 and inhibits breast tumorigenesis by interfering with proliferation and survival signaling. Oncogene 32: 3306-3310, 2013.

24. Jiang Q, He M, Guan S, Ma M, Wu H, Yu Z, Jiang L, Wang Y, Zong $\mathrm{X}$, Jin $\mathrm{F}$ and Wei M: MicroRNA-100 suppresses the migration and invasion of breast cancer cells by targeting FZD-8 and inhibiting Wnt/ $\beta$-catenin signaling pathway. Tumour Biol 37: 5001-5011, 2016.

25. Steber HS, Gallante C, O'Brien S, Chiu PL and Mangone M: The C. elegans 3'UTRome v2 resource for studying mRNA cleavage and polyadenylation, 3'-UTR biology, and miRNA targeting. Genome Res 29: 2104-2116, 2019.

26. Horimoto Y, Sasahara N, Sasaki R, Hlaing MT, Sakaguchi A, Saeki H, Arakawa A, Himuro T and Saito M: High FOXA1 protein expression might predict late recurrence in patients with estrogen-positive and HER2-negative breast cancer. Breast Cancer Res Treat 183: 41-48, 2020.

27. Fu X,Pereira R, De Angelis C, Veeraraghavan J, Nanda S, Qin L, Cataldo ML, Sethunath V, Mehravaran S, Gutierrez C, et al: FOXA1 upregulation promotes enhancer and transcriptional reprogramming in endocrine-resistant breast cancer. Proc Natl Acad Sci USA 116: 26823-26834, 2019.

28. Jing X, Liang H, Hao C, Hongxia L and Cui X: Analyses of an epigenetic switch involved in the activation of pioneer factor FOXA1 leading to the prognostic value of estrogen receptor and FOXA1 co-expression in breast cancer. Aging (Albany NY) 11: 7442-7456, 2019.

29. Petrelli A, Bellomo S, Sarotto I, Kubatzki F, Sgandurra P, Maggiorotto F, Di Virgilio MR, Ponzone R, Geuna E, Galizia D, et al: MiR-100 is a predictor of endocrine responsiveness and prognosis in patients with operable luminal breast cancer. ESMO Open 5: e000937, 2020.

This work is licensed under a Creative Commons Attribution-NonCommercial-NoDerivatives 4.0 International (CC BY-NC-ND 4.0) License. 